Correo: innova@uide.edu.ec

\title{
Emprendimiento en América Latina. Espejismo o realidad
}

\section{Entrepreneurship in Latin America. Mirage or reality}

Juan Carlos Aguirre

Universidad de Cuenca, Ecuador

Autor por correspondencia: juan.aguirreq@ucuenca.edu.ec

Fecha de recepción: 21 de febrero de 2018 - Fecha de aceptación: 15 de agosto de 2018

\begin{abstract}
Resumen
En la actualidad el emprendimiento ha formado parte de un sin número escenarios de análisis, debate, investigación y hasta discursos políticos en pro de su importancia para el desarrollo de los países y su invaluable aporte hacia el fortalecimiento de la clase media. Es así que los aportes realizados sobre esta temática han sido varios, todos coincidentes en la representatividad de construir un ecosistema emprendedor que permita el fomento direccionado, correcto y en las mejores circunstancias posibles con el afán de que aquellos "negocios" recientemente formados coadyuven a mejorar la calidad de vida de las personas en términos macroeconómicos. Razón por la cual en la región se han adoptado medidas diversas que favorezcan la actividad empresarial, muchas de ellas sin lograr aún su objetivo. Es así, que este artículo presenta un análisis descriptivo del estado real del emprendimiento en América Latina, su relación con el crecimiento económico y la incidencia de esta nueva ola de política pública en favor de la actividad emprendedora.
\end{abstract}

Palabras Claves: emprendimiento; crecimiento económico; América Latina; realidad

\begin{abstract}
At present, entrepreneurship has been part of a number of scenarios of analysis, debate, research and even political speeches in favor of its importance for the development of countries and its invaluable contribution to the strengthening of the middle class. Thus, the contributions made on this subject have been several, all coinciding in the representativeness of building an entrepreneurial ecosystem that allows targeted development, correct and in the best possible circumstances with the aim that those newly formed "businesses" help to improve the quality of life of people in macroeconomic terms. Reason why in the region have adopted diverse measures that favor the business activity, many of them without achieving yet to achieve its objective. Thus, this article presents a descriptive analysis of the real state of entrepreneurship in Latin America, its relationship with economic growth and the impact of this new wave of public policy in favor of entrepreneurial activity.
\end{abstract}

Key words: entrepreneurship; economic growth; Latin America; reality 


\section{Introducción}

La relación entre el emprendimiento y el crecimiento económico en América Latina recoge una variabilidad de perspectivas e intenciones de fomento al emprendimiento; determinar el estado real del emprendimiento en América Latina en relación a su aporte al engrandecimiento de las economías de la región.

\section{Estado del arte}

Lundstrom y Stevenson (2001) señalan: "Emprender es perseguir la oportunidad más allá de los recursos que se controlen en la actualidad". Un emprendedor es portador de ideas, innovación, apetito por el riesgo, visión, predisposición a penetrar en nuevos mercados y capacidades académicas y prácticas que colaborarán para que los proyectos en los que se incluya puedan tener mayor probabilidad de éxito.

Desde una perspectiva inicial se puede indicar que el emprendimiento innovador es uno de los principales motores de expansión de la economía, pues impulsará los niveles económicos hacia el desarrollo; arista compartida por los principales economistas clásicos a lo largo del tiempo como: Adam Smith, Jhon Stuart Mill o David Ricardo.

También es cierto que el éxito en el emprendimiento no solamente dependerá del cúmulo de actitudes, capacidades y aptitudes descritas anteriormente sino también de los factores del entorno en donde se desarrollan o se piensan desarrollar los proyectos de emprendimiento, razón por la cual muchos Estados y sus Gobiernos han determinado prudente el auspicio y fomento del emprendimiento en las economías locales, en base a una serie de incentivos y marcos normativos generados específicamente con esta finalidad.

El emprendimiento es comprendido como un fenómeno práctico, que los empresarios experimentan directamente en sus actividades y funciones; como un acto de superación y mejoramiento de las condiciones de vida de la sociedad. (Rodríguez, 2009), es dinámico y quizás la delimitación de sus atributos sea un error al momento de describirlo. Sí podemos relacionarlo con innovador, flexible y creativo (Castillo, 1999 ). Desde la inclusión del emprendimiento economía en la vida empírico-científica, a través de la contribución de los economistas clásicos y neoclásicos (Toledano, 2002) y uno de sus precursores como Jopep Schumpeter (1934), la idea de emprendimiento ha generado cada vez más curiosidad, al estar ligado al crecimiento económico de las naciones. Kirzner, Fritsch, Lucas, Baumol, Van Stel, etc. Su evolución ha sido correspondiente con las formas administrativas, sus contextos, economías globalizantes y en general todo el entorno dinámico construido por las sociedades, resaltando sobre todo la heterogeneidad emprendedora (Crecente, 2009). Es así que el emprendimiento cobra fuerza, y desde hace dos décadas atrás aproximadamente, ya no solamente calificaba a un ciudadano (emprendedor) sino marcaba diferencia con el emprendimiento corporativo (Burns, 2001), lo que le trajo consigo representatividad a nivel empresarial, factor que dio paso, por ejemplo, a las aceleradoras empresariales existentes hoy, una figura de desarrollo de proyectos a través del financiamiento de alguna empresa interesada en potencializar su desarrollo e innovación.

La investigación se la realizará esencialmente para discutir soluciones, posibles puntos falentes, y generar mejores prácticas que encausen el emprendimiento en torno al crecimiento 
económico, apartando la subjetividad y juicios arbitrarios que no contribuyen en nada sobre el tema. Se analizará de forma íntegra las condiciones, entorno y estado de los emprendedores, con la finalidad de concentrar información académico-científica relevante sobre el impacto que ha tenido el emprendimiento en la región.

Es digno de exponer la importancia en la investigación sobre emprendimiento y la literatura científica y académica que lo relaciona o no con el crecimiento económico, pues ayudará sin duda alguna a mejorar las condiciones presentadas por los actores de este segmento económico, delineando de mejor manera sus proyectos y a la postre repercutiendo en el desarrollo económico del país.

\section{El emprendimiento y el crecimiento económico}

Existe variedad de referencias y pensamientos científicos y académicos respecto si el emprendimiento es un factor que se relaciona de alguna forma o mediante algún factor con el crecimiento económico de un país, o si realmente ayuda a su desarrollo o no. Se entiende interesante y trascendental estudiar esta supuesta relación, pues una gran parte de literatura científica da por entendido y como un supuesto casi aceptado globalmente que esta relación es positiva, sin embargo existen quienes tienen un punto de vista contrario y anexan la relación existente a una gama de factores identificados y además a las diversas situaciones económicas de un país, situación analizada en el contexto empírico.

En la actualidad existe gran interés por el emprendimiento relacionado con el desarrollo económico y como parte de este último, el crecimiento económico (Van Stel et al., 2005), situación que hace evidente su trascendencia, por lo que autores como Toca (2010), citado por Somarriva, 2015) señalan: El emprendimiento "constituye un tema en auge, por lo que los gobiernos intentan promoverlo, los individuos aspiran practicarlo, las organizaciones buscan recapturarlo y la academia aspira estudiarlo".

La actividad emprendedora actúa a nivel macroeconómico siendo el motor primordial de creación de empleo, y a nivel micro promueve de forma constructivista el fomento de la empresa y su expansión (Stam, 2007). De esta forma se crea un círculo virtuoso entre economía y empresa que genera mejoramiento económico y fortalecimiento de la clase media, hecho por el cual una economía puede trascender hasta el anhelado status calificado como nación desarrollada.

Existen otros investigadores que manifiestan o incluyen la causalidad positiva entre las dos variables señaladas, apoyándose en evidencia empírica como Acs et al. (2012), otros defienden la dependencia existente entre el emprendimiento y el ciclo económico en el que se lo analiza (Fritsch et al., 2015; Scholman et al., 2015) u otros como Amorós et al., (2012); Galindo y Méndez, (2014); Aparicio et al., (2015), defienden la reciprocidad entre las variables. (Almodóvar, 2016).

En concordancia con lo mencionado se han identificado al menos 3 corrientes que se refieren al emprendimiento y crecimiento económico, explicando como un factor diferencial (causa de crecimiento) que podría ser relacionada y servir para explicar conceptualidades relacionadas al emprendimiento. 
$\checkmark$ Enfoque 1.- Teoría del crecimiento Exógeno (West et al. (2008): capital y trabajo.

$\checkmark$ Enfoque 2.- Teoría del crecimiento Endógeno Plummer y Acs (2014): capital humano y el conocimiento.

$\checkmark$ Enfoque 3.- Nueva Economía Institucional Aparicio et al. (2015): normas e instituciones. (Almodóvar, 2016)

Con estos enfoques se puede concluir que cada una de las corrientes con sus respectivos investigadores, de acuerdo a su postura realizarán el estudio del emprendimiento y su relación con el crecimiento económico basados en factores relacionados a las variables y estas subsumidas a aquella corriente como lo señala Almodóvar (2016): Los investigadores basados en la Teoría Endógena utilizarán variables como la educación y capacitación; los institucionalistas relacionadas con normas institucionales (Urbano y Álvarez, 2014); los basados en Nueva Geografía Económica se valdrán de factores demográficos y poblacionales (Van Oort y Bosma, 2013).

Sin embargo de aquella diversificación de criterios para la utilización de variables, no se puede negar que existen algunas como el PIB, o el progreso tecnológico o el indicador de innovación en un país que irá formando parte transversal de los estudios por su trascendencia en este tema de investigación.

Con todo esto nace una serie de interrogantes ¿Es el emprendimiento la clave para el desarrollo económico? Y de ser así ¿Qué esperan los países para enfocar todos sus esfuerzos en pro de esta variable?, la respuesta es compleja, lo que es seguro es que el emprendimiento es un factor importante y posee relación con el crecimiento económico (Almodóvar, 2016), sin embargo este factor actúa en medio de un proceso, este proceso en un ambiente y este en un contexto. Cada uno de ellos complejos y dinámicos, situación que lleva a generar un sin número de distorsiones importantes respecto esta relación.

América Latina (AL) en los últimos años, ha sido escenario de una serie de acontecimientos, generando interés entre la colectividad internacional, al mostrar un vigoroso crecimiento durante un periodo de tiempo considerable, a pesar de aquello hoy vive una desaceleración generalizada en la región ocasionando dudas sobre su sostenibilidad económica (Banco Mundial, 2016).

Hay quienes consideran que América Latina ha tenido una débil, por decirlo de alguna manera, creación de empresas, pues existe gran cantidad de empresas pequeñas en relación a grandes, o un número similar de empresas con más de 26 años de antigüedad entre esas pequeñas y grandes, situación que según el CAF (2013), indica la falencia y el poco desarrollo en torno al emprendimiento en AL, pues manifiestan que las empresas más exitosas serán las que posean mejores tecnologías, más innovación, acceso a nuevos mercados de manera más sencilla, mejoras en producción, etc., Por lo que las empresas recientemente creadas y además micro, pequeñas o medianas, en términos relativos no aportan mayoritariamente al mejoramiento de estos indicadores. Se debe considerar además, que el bajo nivel de creación e innovación en la región, 
frente a economías desarrolladas (CAF, 2013), sus motivos y demás factores que al momento de emprender forjen un ecosistema desfavorable y poco atractivo para ejercitar una aspiración.

Continuando, Hopenhayn y Neumeyer (2004) y Sanguinetti y Villar (2012) indican explícitamente la poca evolución del PIB en América Latina, y su fundamentación en la falta del mejoramiento de la actividad productiva a través del capital físico y el humano, para que la economía pueda rendir más y expandir su tamaño. Con este enfoque productivo y de generación de valor para incrementar la productividad, surge la relación con la creación de aquellas empresas y su utilidad para el incremento del tamaño económico en un país Thurik, (2008). Por su lado, fortaleciendo la inexactitud de la relación y además dejando el tema inconcluso de cierta forma Liñán y Fernández-Serrano, (2014), manifiestan la falta de modelos que verifiquen y propongan claramente la relación existente, dejándolos poco claros e insatisfactorios.

La región ha sido una de las geografías en las que se ha puesto más atención al emprendimiento y a las condiciones económicas; muchos estudios atribuyen la falta de crecimiento económico y de emprendimiento a aptitudes o información asimétrica o mayor cantidad de emprendimientos solamente de familias con un amplio patrimonio, y también se menciona a las fallas del Estado como el de dar excesivo apoyo a microempresas o empresas pequeñas siendo estas solamente capaces de subsistir y dar empleo a una o dos personas o máximo al entorno familiar, mientras que empresas con características expansivas, mucho más grandes son progresivamente limitadas como producto de las políticas públicas, sin embargo de todo esto, no se ha podido concluir con exactitud el impacto que ha ocasionado este comportamiento.(Konrad-SOPLA, 2016)

La economía latinoamericana en relación al emprendimiento ha sufrido un lento crecimiento, producto, como indica la CAF (2013), de la informalidad y baja productividad como consecuencia del tamaño y estructura de los emprendimientos, generalmente pequeños y además con poco valor agregado. Por su parte las economías desarrolladas no tienen este comportamiento, pues la presencia de las firmas grandes con estructuras adecuadas a sus necesidades y como punto principal de su funcionamiento la competitividad y productividad, encuentran mucho más viable su crecimiento, es decir la relación es positiva respecto el PIB per cápita. Razones por las que la región sea considerada como un laboratorio bastante interesante de investigación en emprendimiento y sus posibles respuestas.

Todas aquellas situaciones formarán parte del análisis planteado, con sus diferentes posiciones y alcances, considerando las variables y factores que en base a esas perspectivas tengan la intencionalidad de medir el emprendimiento en relación al crecimiento económico. El investigador Stenholm et al., (2013), indica la trascendencia de las variadas formas de cuantificar el emprendimiento indicando a las disparidades que se llegará en torno a los criterios y demás información que se utilice.

\section{Metodología}

El presente documento de investigación realiza un análisis exploratio de la bibliografía más representativa entre el emprendimiento y el crecimiento económico en América Latina, con 
un un foque descriptivo sobre la información obtenida por el Banco Mundial BM), Banco de Desarrollo de América Latina (CAF), CEPAL y General Entrepreneurship Monitor (GEM).

El BM, CAF y CEPAL son organizaciones multinacionales, que realizan estudios sobre una amplia gama de variables tendientes a explicar la economía, aspectos culturales, políticos, sociales etc., en muchos países nivel global. Cuentan con bases históricas y reportes generados que permiten un análisis pormenorizado de la información generada.

Por su parte General Entrepreneurship Monitor, cuenta con una metodología aplicada con el mismo criterio a las economías en donde practica el estudio. Los resultados se obtienen a través de encuestas realizadas a dos grupos poblacionales entre los 18 y 93 años de edad. Los primeros son una muestra representativa de todo el conglomerado y además otro grupo encuestado correspondiente a los expertos en la materia de emprendimiento.

\section{Resultados}

\section{El emprendimiento y el crecimiento económico en la región}

Para el análisis del emprendimiento, existe una variabilidad de información importante respecto el enfoque que se pretenda esquematizar. Al analizar los resultados más representativos para el emprendimiento relacionado con el crecimiento económico se cuenta con organizaciones reconocidas a nivel mundial. Las cuales podrán aportar con datos en concreto de los hechos actuales, como el Banco de Desarrollo de América Latina, Banco Mundial, CEPAL, y por su puesto General Entrepreneurship Monitor (GEM).

Al analizar los datos expuestos por el Banco mundial respecto a la economía Latinoamericana, tenemos que la región alcanzó un 3,2\% de crecimiento económico promedio entre 2000 y 2014, situación favorable sin embargo podemos verificar un estancamiento abrupto de ahí hacia la actualidad, principalmente atribuido a la disminución de precio de las materias primas, que para algunas economías de la región son su pilar fundamental. La clase media aún no se encuentra constituida, teniendo al $40 \%$ de población en promedio aún en las clases vulnerables, ocasionando que se mantengan las brechas tan amplias respecto los estratos y clases sociales en la región.

Las empresas medianas y grandes tienen mayor posibilidad de innovar, además pagan un salario entre 20 y $40 \%$ mayor a las pequeñas empresas (Lederman et al., 2014), mismas que coadyuvan para el mejoramiento económico en conjunto de forma significativa, sin embargo existen economías como la ecuatoriana en la que las empresas familiares, generalmente micro y pequeñas bordean el $85 \%$ del total de empresas en la región, siendo estas de bajas expectativas de crecimiento acelerado. Situación que marca la situación que viven las economías emergentes o en vías de desarrollo.

Los emprendimientos latinoamericanos reportan que casi dos tercios de la población presenta actitudes sociales positivas sobre este factor (GEM, 2015), además de un grupo de emprendedores nacientes del 13\% superior a otras regiones analizadas. Sin embargo pese a este escalonamiento en "empresas" la tasa de crecimiento latinoamericano en 2014 fue de $-1,1 \%$ y en 
el 2015 apenas un crecimiento de 0,5\% (CEPAL, 2015). Situación bastante llamativa, pues los modelos de crecimiento endógeno, muestran la gran importancia de las empresas en el fomento del crecimiento económico, sin embargo la región muestra un comportamiento diferente. Altas tasas de emprendimiento y bajo o casi nulo crecimiento económico en conjunto, esto considerando las heterogeneidades presentes en los países.

Todo lo descrito se encuentra expuesto en el siguiente gráfico, pues en países desarrollados y países en vías de desarrollo el comportamiento del tamaño de su economía es diferente:

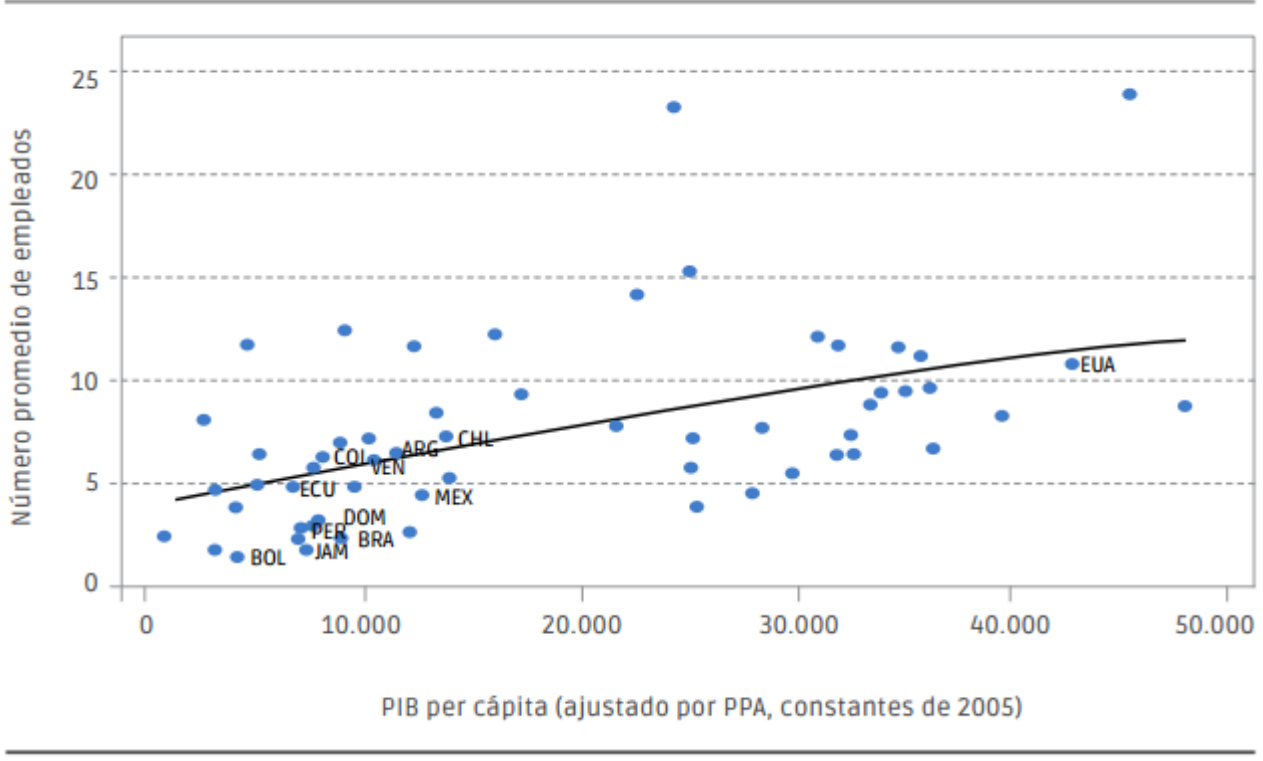

Gráfico 1.- Tamaño de las empresas y nivel de desarrollo Fuente: CAF 2013 - GEM (2012)

Como se verifica existe más presencia de firmas grandes en economías desarrolladas que firmas grandes en economías en vías de desarrollo con total independencia del sector económico que se considere. Además de su correspondencia con el crecimiento de cada una de esas economías.

El alto grado de inestabilidad económica, apoyado por la falta de empleo han generado un inusual panorama de emprendimiento empresarial en América Latina, con resultados como los señala la investigación hecha por GEM 2015 como el 46\% de encuestados que tienen la idea de emprender sin embargo no la concretan. El Ecuador lidera los emprendimientos en América Latina, sin embargo hay que considerar que se midió en $24,5 \%$ a negocios nacientes y tan solo el 9,9\% a negocios que han operado por 3 años. Los activos de 3 a 6 años son el $5 \%$ y los que llegan a 6 y 9 años son el 2\%. (GEM, 2015).

El emprendimiento naciente en los países de América Latina ha sido significativamente importante, ocasionando que algunos de los países de la región figuren como el top 10 de los más emprendedores a nivel mundial, hecho que ha generado controversia y estudios analíticos sobre 
los factores que ocasionaron este resultado y además los impactos sobre la economía de esos países.

Otro dato importante señalado por el GEM es que en América Latina el 43\% de emprendedores esperan generar al menos un puesto de trabajo, lo que guarda competa relación con el tipo de emprendimiento predominante en la región; empresas pequeñas que generalmente se crean para satisfacer un salario necesario para la subsistencia de aquellas personas, situación diferente se vive en Chile logrando que el 50\% de sus emprendedores cuentan con innovación (GEM, 2015), una más de las razones para que este país sea el referente general para muchos países de LAC.

\section{El emprendimiento y el crecimiento económico en el Ecuador}

El Ecuador se ubica como se dijo, en el año 2015 como el primer país más emprendedor de la mencionada región, estando delante de Chile, Colombia, Perú, etc., según estudio realizado por GEM 2015, llegando a una Actividad Emprendedora Temprana de 33,6\%, situación que desde una perspectiva positivista podría sugerir un avance para la economía nacional en términos pro activos, responsables, eficientes e innovadores y coadyuvantes para el tan publicitado, "Cambio de la Matriz Productiva Ecuatoriana".

Sin embargo también se puede deducir que el marco del emprendimiento en el Ecuador, cuenta con distorsiones respecto su objetivo final, tratadas en asuntos sociales, culturales políticos y económicos, como la generación de emprendimientos basados en la necesidad y no en la oportunidad, es decir creadas en gran medida por las dificultades económicas y las restricciones de oferta laboral en el país y no impulsadas por la tecnología, la innovación y el entregar el valor agregado a sus productos o servicios para tener mayor probabilidad de establecerse en el tiempo.

Llevados a las preguntas ¿Por qué con este gran número de emprendimientos, la economía se encuentra estancada y muchos casos en detrimento? Si los emprendimientos forman parte del crecimiento económico de un país ¿Por qué el Ecuador al ser el país que más emprendimientos presenta en la región, no es el más próspero o el crecimiento de su economía ha sido significativo y sostenido? ¿Por qué no se acaba de consolidar estructuralmente el llamado Cambio en la Matriz Productiva?

Situación que inequívocamente conduce al análisis de la calidad o cantidad de los emprendimientos en el Ecuador y en la región, su relación respecto su crecimiento económico, además a la pertinencia de fomento legal y tributario existente en el país relacionado con el incentivo del emprendimiento, situación bastante curiosa, pues existen al menos 4 regulaciones legales en pro del emprendimiento y varias reformas tributarias, sin embargo gran parte de los emprendedores del país mantienen la tendencia de la insostenibilidad en el tiempo, muchas de las veces atribuido a la falta de diferenciación de productos y a la alta concentración que tienen los emprendimientos comerciales, sin que estos generen mayor valor agregado, contraponiéndose, de cierta manera, a las políticas del Gobierno alineadas con la mejora de la competitividad, diversificación de la productividad y el eje central de la innovación, emprendimientos que no han generado impactos positivos en sentido estricto a la economía ecuatoriana. Surgiendo con esto el 
cuestionamiento a las políticas generadas, su pertinencia y el impacto que han causado, evaluar en términos de calidad, si lo realizado hasta el momento pone al Ecuador en el camino correcto hacia el crecimiento, desarrollo económico y social tan anhelado, o por el contario genera reflexión lo logrado, enfocando a las autoridades de mejor manera, sugiriendo tomar medidas correctivas a tiempo, como políticas más asertivas y pertinentes, con mayor y mejor alcance entre el emprendimiento, la innovación y los objetivos macroeconómicos fomentados por el Estado.

Factores que deberán ser estudiados, relacionando los incentivos otorgados por el estado para promocionar el emprendimiento y los resultados conseguidos por los emprendedores de la ciudad, determinando además factores de afección positivos y negativos y creando conclusiones definidas que orienten de mejor manera esta actividad. Pues aún en una verificación simple entre el emprendimiento y crecimiento económico para algunas de las economías de la región su resultado es desalentador (Véase gráfico 2)

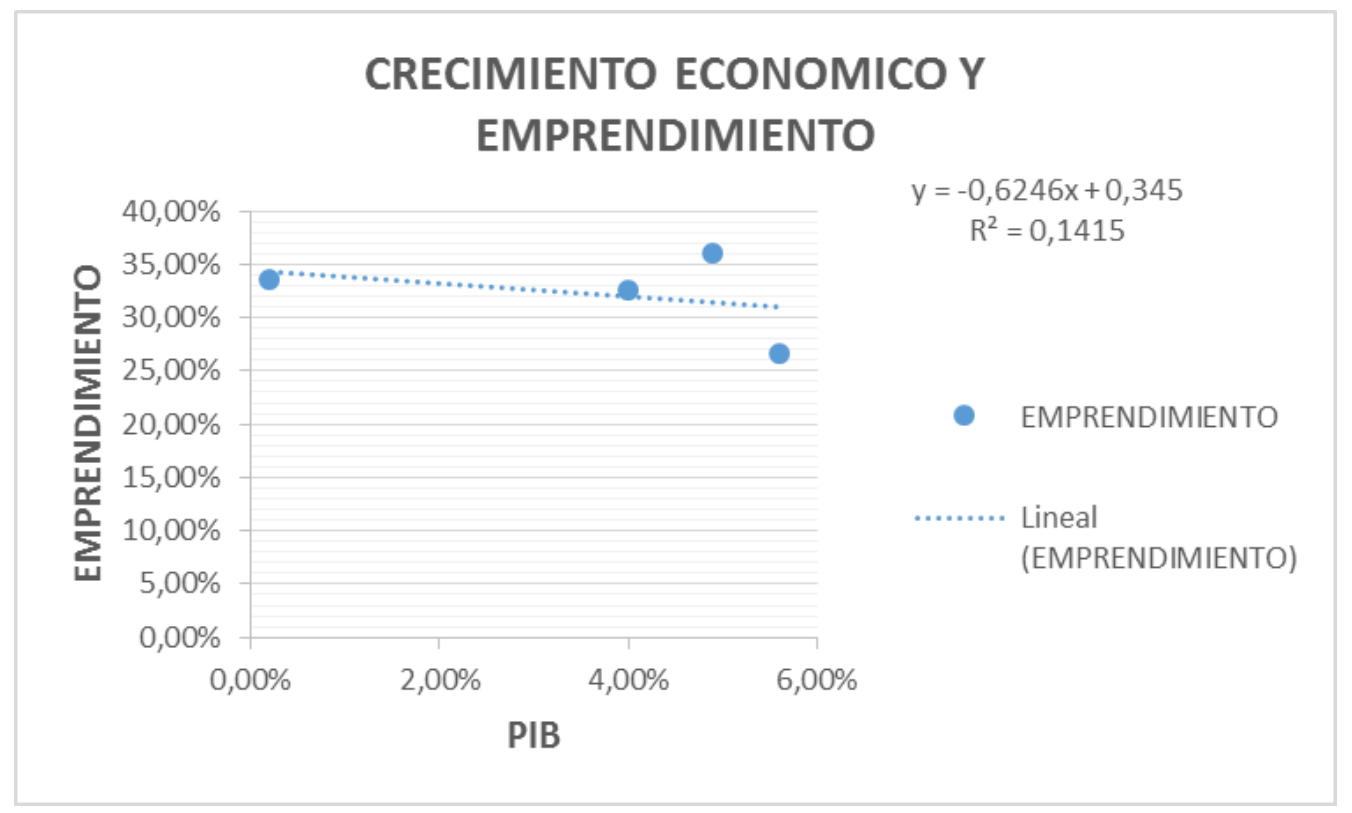

Gráfico 2.- PIB - Creación de empresas Fuente (GEM, 2015); (Banco Mundial, 2015)

Elaboración propia

Los estudios a nivel nacional sobre el tema han traído importantes aportes sobre los puntos de interés de la investigación. GEM expone una realidad preocupante sobre el emprendimiento generado en la región indica que las Economías con mayor emprendimiento no son las más sólidas o desarrollas sino más bien lo contrario, puntualiza que Uganda es el país con mayor emprendimiento en el mundo, y a pesar de aquello, el desarrollo económico reportado es poco o nada significativo con sus comparables a nivel mundial. Por otro lado EEUU posee una TEA menor a la mitad de los índices obtenidos en algunos de los países de América Latina, sin embargo posee una de las economías más importantes del mundo y sólida al consolidar sus emprendimientos.

Situación bastante curiosa e importante analizarla, pues el emprendimiento es tratado como una de los caminos más plausibles contra el subdesarrollo, sin embargo el tratamiento y la 
realidad muestra que dependerá de la calidad con la que se maneje esta variable y sobre una supuesta causalidad entre el emprendimiento y el crecimiento económico.

\section{Discusión}

Las economías de algunos países de América Latina y el Caribe han estado en el ojo del análisis de importantes inversionistas y empresarios a nivel mundial, pues a lo largo de algunos años han mostrado niveles de crecimiento económicos acelerados y sostenidos (Banco Mundial, 2015). Comportamiento frenado en los últimos tiempos por la crisis proveniente de el decremento marcado sobre sus comodities, baja de materias primas, competitividad y esencialmente sobre la actividad extractivita petrolera, y por nombrarlo, acentuado en el Ecuador por su nula política monetaria, creando una serie de dudas respecto a lo logrado hasta la fecha, desestabilizando la imagen ex abrupta de crecimiento.

Durante los años en los que la región, en su gran mayoría, ha sido gobernada por líneas izquierdistas, socialistas o progresistas, como se las desee denominar, se han visto claramente objetivos ligados a las políticas públicas paternalistas encaminados a mejorar las condiciones de vida de sus habitantes, siendo prioritario en el orden del día normativas, leyes, decretos, políticas y acciones dirigentes a mitigar la pobreza; disminuir la brecha existente entre los polos de las clases sociales-económicas y además impulsar la economía, muchas de las veces recurriendo excesivamente al presupuesto público. Actos que tuvieron impacto positivo en la región, durante algún tiempo como lo indican Daniel Lederman y otros en su libro publicado en el año 2014 titulado "El emprendimiento en América Latina: Muchas empresas poca innovación:

- Casi 70 millones de personas salieron de la pobreza en la última década.

- Aproximadamente 50 millones de personas se unieron a la clase media entre 2003 y $2009 \ldots$ (Lederman et al., 2014)

Uno de los problemas de mayor envergadura a nivel regional y nacional se visualiza en el desempleo, subempleo o empleo inadecuado, razón por la cual se genera un comportamiento volcado hacia la generación de autoempleo, situación que ha cobrado una fuerza por llamarla desmedida y desorientada en el contexto generalizado de las economías emergentes de América Latina fenómeno de gran interés para el estudio y análisis. Como bien lo indica Stam et, al (2007), el emprendimiento es una oportunidad para mejorar las circunstancias actuales. En este contexto a continuación la tasa de desempleo en la región, en donde se verifican porcentajes significativamente importantes, pudiendo ser la razón, quizás de fondo para el escalonamiento del emprendimiento. (Véase tabla 1)

Tabla 1. Tasa Desempleo en América Latina y el Caribe

\begin{tabular}{rrrrrrrrr}
\hline \multicolumn{1}{c}{ Año } & $\mathbf{2 0 1 0}$ & $\mathbf{2 0 1 1}$ & $\mathbf{2 0 1 2}$ & $\mathbf{2 0 1 3}$ & $\mathbf{2 0 1 4}$ & $\mathbf{2 0 1 5}$ & $\mathbf{2 0 1 6}$ & $\mathbf{2 0 1 7}$ \\
\hline $\begin{array}{l}\text { Te } \\
\text { desa }\end{array}$ & 7.634 & 7.155 & 6.801 & 6.664 & 6.628 & 7.111 & 8.142 & 8.769 \\
\hline
\end{tabular}

Fuente: Banco Mundial, (2017) 
Se verifica además, que las naciones Latinoamericanas han hecho las cosas bien respecto a un sin número de situaciones sociales, económicas, empresariales, etc., sin embargo actualmente existe un estancamiento (GEM, 2015). Por su parte, el Ecuador es uno de los países más emprendedores dentro de América Latina según estudios realizados por Global Entrepreneurship Monitor (GEM, 2015), llegando a niveles de emprendimiento superiores a Colombia, Chile, Uruguay, incluso Brasil. Situación, por decirlo menos llamativa, al estar catalogado como un "un país en vías de desarrollo". Lo que lleva a pensar en la sostenibilidad de una empresa en este contexto, de su participación activa en la innovación y su adaptabilidad en el ambiente en el que se desarrolla. Constant y Zimmermann (2006), son de los que piensan que "El emprendedor siempre debe tener una visión a largo plazo basada en relaciones personales". ¿Causa, quizá, para que en América Latina exista esta volatilidad de resultados, el emprender pensando en una medida coyuntural de actividad económica que permita, a su fundador, contar con un back up, mientras se enrola nuevamente al servicio público? Situación que abre nuevos frentes de investigación.

La sostenibilidad empresarial muestra que los países menos sostenibles empresarialmente son Bolivia y Nicaragua con aproximadamente 1.67\% del total. Ecuador queda en un "honroso" sexto lugar.

Ahora, se debe tener presente la diferencia sustancial entre las economías Latinoamericanas, y quizás la política que adopte una nación no necesariamente aporte valor en otra. Razón por la cual se ha palpado un abanico extenso de medidas tomadas para aprovechar este "factor" de emprendimiento en pro de las economías, así tenemos que algunas regulaciones creadas por los estados para apoyar la actividad emprendedora en su geografía como: en Argentina se creó la ley 24.267 y la ley 25.300 ligadas al fomento del emprendimiento; en Brasil la ley complementaria 123(2006); en Chile Ley 20.416 (2010); en Colombia la ley 590 (2000); y, así por el estilo gran parte de la comunidad regional ha visto una oportunidad en el emprendimiento, coherente con las circunstancias actuales y las metas que persiguen. Más, no se puede dejar de considerar el contenido variable de esta normativa, pues la política pública será a la medida, es decir generar alternativas y oportunidad en virtud de sus particularidades, como bien lo explica Dana (2010) indicando que no se trascriben políticas de éxito que ha tomado otro país, pese a su similitud que puedan tener.

Emprendimiento en América Latina ¿realidad o no?, los resultados arrojan disparidades en torno al crecimiento económico y también al emprendimiento, lo que es cierto es que se ha verificado un ambiente para emprendimiento no muy favorable en algunas naciones, en especial las que cuentan con un marco regulatorio variable, provocando inseguridad jurídica y repercutiendo directamente sobre el riesgo evaluado por inversionistas al momento de montar una empresa. Como se ha expresado generalmente el emprendimiento, correctamente establecido, podría ser la clave para el mejoramiento de la calidad de vida de las personas radicas en un lugar geográfico. Sin embargo se puede colegir los intentos aislados que generan los países, con el afán de mejorar las condiciones de emprendimiento, pero no dejan de ser esfuerzos aislados, sin que estos provoquen un cambio estructural en el emprendimiento y su manera de planificarlo, financiarlo y hasta ejecutarlo. Pues se sigue la corriente y lastimosamente existe muchas empresas y poca innovación (Lederman et al., 2014) 


\section{Conclusiones}

Concluyendo el tema podemos decir que los limitantes del emprendimiento en Latinoamérica son varios, destacando entre ellas la escasa educación y formación de emprendimiento, así como las altas cargas impositivas y el apoyo aislado que resulta insuficiente por parte del estado.

Del análisis se puede manifestar que existen condicionamientos fuertes y arraigados en el costumbrismo al momento de emprender, es mucho más difícil hacer las cosas de manera diferente.

A pesar de contar con altas tasas de emprendimiento en la región, se puede manifestar que este no se encuentra bien concebido, o al menos podría ser mejor. Siendo una de las respuestas para los emprendedores concientizados y con visión sostenible, la innovación.

Las políticas públicas generadas en torno al emprendimiento son distintas, y está bien, sin embrago deberían focalizar los esfuerzos estatales por incluir aquellas en un aparataje más grande, en un proceso plenamente estructurado capaz que cause sinergia en los resultados que puedan conseguirse a partir del fomento al emprendimiento.

Para terminar, también es prudente señalar el impulso a la competitividad, misma que podría lograrse a través de una apertura de las fronteras con acuerdos internacionales, que obliguen a las empresas originarias de un país, a volverse competitivas.

\section{Bibliografía}

Acemoglu, D. \& Robinson, J.A. (2012). Why Nations Fail. New York: Crown

Acs, Z.J. \& Amorós, J.E. (2008). Entrepreneurship and Competitiveness Dynamics in Latin America. Small Business Economics

Acs, Z.J.; Audretsch, D.B.; Braunerhjelm, P. \& Carlsson, B. (2012). Growth and entrepreneurship. Small Business Economics

Acs, Z.J.; Szerb, L. \& Autio, E. (2015). Global Entrepreneurship Index 2015. Washington, D.C.: The Global Entrepreneurship and Development Institut.

Álvarez, C.; Urbano, D. \& Amorós, J.E. (2014). GEM research: achievements and challenges. Small Business Economics

Anokhin, S. \& Wincent, J. (2012). Start-up rates and innovation: A cross-country examination. Journal of Business Venturing. 
Amorós, J.E; Fernandez, C. \& Tapia, J.(2012). Quantifying the relationship between entrepreneurship and competitiveness development stages in Latin America. International Entrepreneurship and Management Journal,

Almodóvar, M. 2016. Actividad emprendedora y crecimiento económico.

Aparicio, S.; Urbano, D. \& Audretsch, D.B. (2015). Institutional factors, opportunity entrepreneurship and economic growth: Panel data evidence. Technological Forecasting \& Social Change, Próximamente. Disponible en: http://www.sciencedirect.com /science/article/pii/S0040162515000992.

Audretsch, D.B. (2012). Entrepreneurship research. Management Decision

BCE. (2015). Banco Central del Ecuador. Recuperado el 1 de Diciembre de 2015, de Cifras Económicas de Ecuador: http://www.bce.fin.ec/index.php/component/k2/item/ 312- cifrasecon\%C3\%B3micas-del-ecuador

Banco de Desarrollo para América Latina (CAF). 2013. Investigación para el desarrollo. Obtenido de: https://www.caf.com/es/temas/i/investigacion-para-el-desarrollo/

Banco Mundial. (2016). Data Banco Mundial. Obtenido de: http://www.bancomundial.org

Ben Letaifa, S. \& Rabeau, Y. (2013). Too close to collaborate? How geographic proximity could impede entrepreneurship and innovation. Journal of Business Research

Burns, P. (2001). “Entrepreneurship and Small Business”, Palgrave, Great Britain.

Castillo, A. (1999). "Estado del Arte en la Enseñanza del Emprendimiento", First Public Inc., Chile, S.A.

CEPAL. (2015). Estudio económico de América Latina y el Caribe 2015.

CEPAL. (2017). Datos y estadísticas. Obtenido de: https://www.cepal.org/es/datos-y-estadisticas

Constant, A. y Zimmermann, K (2007). Measuring ethnic identity and its impacto n economic behaviur.

Cordero López, J. (2011). Universidad de Cuenca.Obtenido de Análisis de los factores que influyen el emprendimiento y la sostenibilidad de las empresas del área urbana de la ciudad de Cuenca, Ecuador

Crecente, F. (2009). “Análisis de la financiación de la actividad emprendedora”, Tesis doctoral, Universidad de Alcalá, CEMPRESA.

Dana, L. (2010). “Entrepreneurship and Religion”, Edward Elgar Publishing Limited, USA. 
Daniel, L.; Messina, J; Pienknagura, S; Rigolini, J. 2015. El Emprendimiento en América Latina: Muchas empresas y poca innovación.

Escuela de Negocios Politécnica del Litoral. (2014). Escuela de Negocios Politécnica del Litoral. Obtenido de Global Entrepreneurship Monitor: http://www.espae.espol. edu.ec /images/documentos/publicaciones/libros/gemecuado r2014.pdf

Fritsch, M; Kritikos, A; Pijnenburg, K. (2015). Business clicles, unemployment and entrepreneurial entry-evidence from Germany,

Galindo, M. 2010. "Entrepreneurship, crecimiento económico y ética”, Estudios de Economía Aplicada

Galindo, M; Méndez, M. (2014). "Entrepreneurship, economic growth and innovation: Are feedbacks effects at work?. JOurnal and Business Research.

GEM. (2014). Global Enterpreneurship Monitor.Obtenido de Global Enterpreneurship Monitor Ecuador 2014 http://www.espae.espol.edu.ec/images/documentos/ publicaciones /libros/gemecuador2014.pdf

GEM. (2016). GEM América Latina y el Caribe 2015/2016.

GEM. (2017). Global Entrepreneurship Monitor Ecuador 2016. ESPAE - ESPOL

GEM. (2017). Data entrepreneurial behaviour and attitudes and Entrepreneurial Framework

Conditions. Obtenido de http://www.gemconsortium.org/data

Grupo Banco Mundial. (2015). Grupo Banco Mundial. Obtenido de http://search.worldbank.org/all?qterm=ifc+pymes+ecuador\&title=\&filetype=\#

Hopenhayn, H. y Neumeyer, P (2004). Latin America in the twentieth century: stagnation, then collapse.

INEC. (2010). Instituto Nacional de Estadísitas y Censos. Recuperado el 1 de Noviembre de 2015 de http://www.inec.gob.ec/

Kantis, H., (2009). Programa de desarrollo emprendedor. Descargado de http://www.prodem.ungs.edu.ar el 8 de febrero de 2011.

Konrad-Adenauer-Stiftung e.V. (2016). La fuerza de la innovación y el emprendimiento. Obtenido de: http://www.kas.de/wf/doc/kas_47118-544-1-30.pdf?161121141209

McClelland, D, (1961). The Achieving Society. MacMillan Publ., New York, USA. 
Liñan, F; Fernandez-Serrano, J. (2014). Nacional culture, Entrepreneurship and economic development: different patterns across the Europe Union. Small Business Economics.

Lundstrom, A., y Stevenson, L. (2001). Entrepreneurship Policy for the future. Special Edition. SME Forum 19-20, March 2001. Swedish Foundation for Small Business Research, Växjö, Sweden.

Observatorio Pymes UASB. (2012). Observatorio de las Pymes de la Universidad Andina Simón Bolívar. Obtenido de Pymes Exportadoras: http://portal.uasb.edu.ec/contenido_centro_programa.php ?cd_centro=15

Rodríguez, A. (2009). Nuevas perspectivas para entender el emprendimiento empresarial

Sanguinetti, P., \& Villar, L. (2012). Patrones de desarrollo en América Latina: ¿convergencia o caída en la trampa del ingreso medio? CAF Documento de trabajo, 2012/02, Caracas: CAF. Retrieved from http://scioteca.caf.com/handle/123456789/231

Stam, E. (2007). A contribution to the theory of economics growth. Quarterlly Journal of Economics.

Stenholm, P; Acs, Z \& Wuebker, R. (2013). Explory country-level institutional arrangements on the rate and type of entrepreneurial activivity. Journal of Business Venturing.

Somarriva, F. (2015). Actividad Emprendedora en el Desarrollo Económico Local. Evidencias para el caso de Nicaragua y Municipio de León, 2000-2013.

Scholman, G.; Van Stel, A. \& Thurik, A. (2015). R The relationship among entrepreneurial activity, business cycles and economics openess. Internacional Entrepreneurship Theory and Practice.

Toca, C. (2010). "Consideraciones para la formación en emprendimiento: explorando nuevos ámbitos y posibilidades”, Estudios Gerenciales

Toledano, N. (2002). "La creación de empresas: un estudio empírico sobre los instrumentos de apoyo al emprendimiento en el ámbito rural onubense", Trabajo de investigación, Universidad de Huelva.

Thurik, A. Carree, M, Van Stel, A. Audretsch, D. (2008). Does self-employment reduce unemployment? Journal of Business Venturing.

Van Oort, F. \& Bosma, N. (2013). Agglomeration economies, inventors and entrepreneurs as engines of European regional economic development. The Anals of Regional Science.

Van Stel, A. Carree, M. \& Thurik, (2005). "The effect of entrepreneurial activity on national economic growth", Small Business Economics 
Van Stel, A. \& Storey, D. (2004). "The link between firm births and job creation: Is there a Upas tree effect?", Discussion Papers on Entrepreneurship, Growth and Public Policy, No. 3304, Group Entrepreneurship, Growth and Public Policy, MPI, Jena. 